\title{
A Polystyrene-Supported 9-amino(9-deoxy)epi Quinine Derivative for Continuous Flow Asymmetric Michael Reactions
}

\author{
Javier Izquierdo, a Carles Ayats, a Andrea H. Henseler, a Miquel A. Pericàs* $a, b$
}

A polystyrene (PS)-supported 9-amino(9-deoxy)epi quinine derivative catalyzes Michael reactions affording excellent levels of conversion and enantioselectivity using different nucleophiles and structurally diverse enones. The highly recyclable, immobilized catalyst has been used to implement a single-pass, continuous flow process (residence time: 40 min) that can be operated for 21 hours without significant decrease in conversion and with improved enantioselectivity with respect to batch operation. The flow process has also been used for the sequential preparation of a small library of enantioenriched Michael adducts.

\section{Introduction}

In response to economy and sustainability concerns, the chemical and pharmaceutical industries are paying increasing attention to aspects such as process intensification, waste reduction, and recycling of solvents and catalysts. Continuous flow processing, still largely overlooked by the fine chemicals and pharmaceutical sectors, satisfies many of these industrial requirements and is hence becoming an interesting alternative to inherently less efficient batch processing. ${ }^{1}$ When catalytic flow processes for the production of enantiopure materials are considered, the use of immobilized catalysts ${ }^{2}$ appears as the most promising alternative. Organocatalysts, not requiring metal cofactors and thus delivering reaction products devoid of toxic metal impurities are perfect candidates for this task.

The cinchona alkaloids have been known for over 200 years and have been used in multiple applications. ${ }^{3}$ In particular, the dense functionality of the molecules of these alkaloids has allowed their easy conversion into versatile organocatalysts. Primary amines prepared in this way, like the 9-amino(9deoxy)epi cinchona alkaloids, have broadened the scope of classical aminocatalysis, traditionally centered on secondary amines such as proline and diarylprolinols. ${ }^{4}$

Cinchona alkaloids and derivatives have been immobilized onto different solid supports like mesoporous silica, ${ }^{5}$ Nylon, ${ }^{6}$ magnetic nanoparticles, ${ }^{7}$ or organic polymers. ${ }^{8}$ However, only a small family of polymer-supported cinchone derivatives, prepared using thiol-ene chemistry, has been briefly evaluated in asymmetric organocatalysis. ${ }^{9}$

In any case, the application of immobilized cinchone derivatives to asymmetric aminocatalysis in flow remains completely unexplored. ${ }^{10}$

\section{Results and discussion}

At the light of the excellent performance depicted by organocatalysts immobilized onto resins through coppercatalyzed alkyne-azide cycloaddition (CuAAC) reactions, ${ }^{11}$ we considered the possibility of using this methodology for the immobilization of cinchona alkaloids. We report in this communication the implementation of this strategy and the use of the resulting functional resins as highly active and enantioselective organocatalysts for a variety of asymmetric Michael additions under batch and flow conditions.

Key to our plans, the vinyl group of the natural cinchona alkaloids in the starting materials had to be converted to an ethynyl group. ${ }^{12}$ With the ethynyl derivatives in hand, CuAAC reactions with azidomethyl polystyrene led uneventfully to polystyrene-supported 9-amino(9-deoxy)epi cinchona alkaloids 1a-4a with high levels of functionalization (from 0.87 to 0.91 $\mathrm{mmol} / \mathrm{g}$. See ESI $\dagger$ ). For comparison purposes the homogeneous analogues $\mathbf{1 b}-\mathbf{4 b}$, resulting from CuAAC reactions with benzyl azide, were also prepared (Scheme 1).

The asymmetric Michael addition is one of the most extended and general methods of C-C bond formation leading to the creation of stereogenic centers. ${ }^{13}$ Among the different strategies for achieving stereocontrol of this process, iminium activation of $\alpha, \beta$-unsaturated carbonyl compound has provided excellent results. ${ }^{13 \mathrm{c}-\mathrm{h}}$ The Michael addition of ethyl nitroacetate (6a) to (E)-4-phenylbut-3-en-2-one (5a) was tested with benchmark catalysts $\mathbf{1 a - 4 a}$, as the densely functionalized products arising from this reaction have found application in synthesis. $^{14}$ 


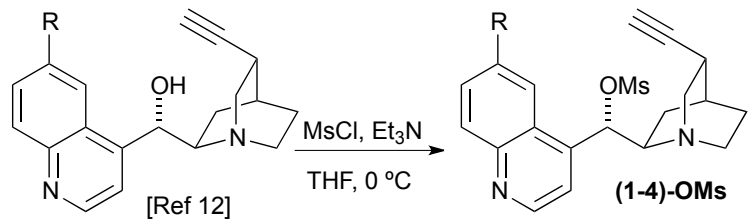

quinine - 1; cinchonidine - 2 quinidine - 3 ; cinchonine -4

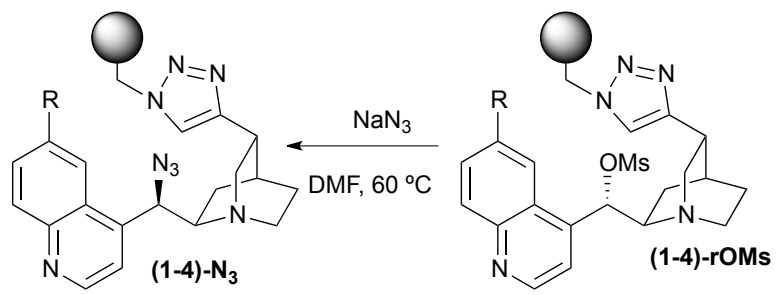<smiles>[R]c1ccc2nccc([C@@H](N)C3CCCCC3c3cn(Cc4ccccc4)nn3)c2c1</smiles>

1a-b, $\mathrm{R}=\mathrm{OMe}$

2a-b, R = H

a series, $Q=(1 \%$ DVB $) P S ;$ b series,$O=P h$

Scheme 1: Synthesis of PS-supported 9-amino(9-deoxy)ep cinchona alkaloids and their homogeneous analogues.

Among the PS-supported cinchona derivatives 1a-4a, quinine derivative 1a, gave the highest enantioselectivity. Likewise, the homogeneous quinine derivative $\mathbf{1 b}$ also showed the best performance in terms of enantioselectivity, with results comparable to those obtained with resin 1 a (See ESI $\dagger$ ). We accordingly focused on the optimization of reaction conditions using the heterogeneous quinine derivative 1a as the catalyst. We started the optimization screening solvents with different characteristics. In the initial screening, $\mathrm{CH}_{2} \mathrm{Cl}_{2}$ showed the highest levels of conversion and enantioselectivity (Table 1. entry 2). A polar solvent mixture, such as a $\mathrm{H}_{2} \mathrm{O} / \mathrm{DMF}$ (50:50) mixture (entry 3), or THF (entry 6) afforded lower selectivities and conversions. Moreover, the reaction did not proceed under neat conditions (entry 1), in xylenes (entry 4) or in MTBE (entry 5), probably because of inefficient swelling of the resin in these solvents. The nature of the acidic co-catalyst required for the reaction was also analyzed. CSA or $p$-TSA were ineffective, the formation of reaction product being not observed at all (entry 7). The use of TFA led to partial conversion and decreased enantioselectivities (entry 8) and 2fluorobenzoic acid did not led to any significant improvement compared to benzoic acid (entry 9). Finally, parameters as concentration or temperature were also modified. Increasing or decreasing the $1 \mathrm{M}$ initial concentration led to lower conversions and enantioselectivities (entries 11 and 12). Somewhat surprisingly, by slightly increasing the temperature to $30{ }^{\circ} \mathrm{C}$, we could achieve almost full conversion in the standard reaction time $(5 \mathrm{~h})$ together with a small increase of enantioselectivity (entry 13). Low-power microwave (MW) irradiation $(2 \mathrm{~W})$ was attempted in order to increase the rate of the transformation (entry 14), but without positive effect.

Table 1. Optimization of the reaction conditions.

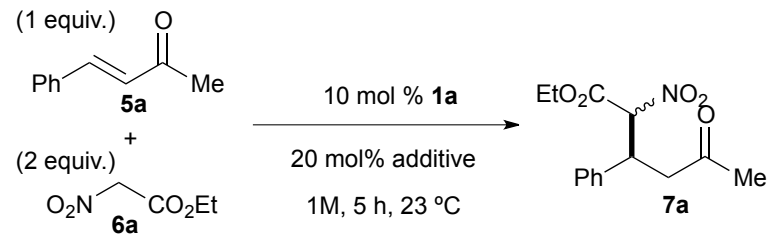

\begin{tabular}{cccccc}
\hline Entry & Solvent & Additive & Conversion (\%) & d.r. $^{\mathbf{a}}$ & ee (\%) \\
\hline 1 & - & PhCOOH & - & - & - \\
2 & $\mathrm{CH}_{2} \mathrm{Cl}_{2}$ & PhCOOH & 91 & $1: 1$ & $94 / 94$ \\
3 & $\mathrm{H}_{2} \mathrm{O} / \mathrm{DMF}$ & $\mathrm{PhCOOH}$ & 83 & $1.1: 1$ & $88 / 89$ \\
4 & $\mathrm{Xylene}$ & $\mathrm{PhCOOH}$ & - & - & - \\
5 & $\mathrm{MTBE}$ & $\mathrm{PhCOOH}$ & - & - & - \\
6 & $\mathrm{THF}$ & $\mathrm{PhCOOH}$ & 33 & $1: 1$ & $82: 90$ \\
7 & $\mathrm{CH}_{2} \mathrm{Cl}_{2}$ & $\mathrm{CSA} \mathrm{or} p-\mathrm{TSA}$ & - & - & - \\
8 & $\mathrm{CH}_{2} \mathrm{Cl}_{2}$ & TFA & 55 & $1.3: 1$ & $81 / 87$ \\
9 & $\mathrm{CH}_{2} \mathrm{Cl}_{2}$ & $2-\mathrm{F}-\mathrm{PhCOOH}$ & 87 & $1: 1$ & $94 / 95$ \\
$10^{\mathrm{c}}$ & $\mathrm{CH}_{2} \mathrm{Cl}_{2}$ & $\mathrm{PhCOOH}$ & 77 & $1: 1$ & $91 / 94$ \\
$11^{\mathrm{d}}$ & $\mathrm{CH}_{2} \mathrm{Cl}_{2}$ & $\mathrm{PhCOOH}$ & 58 & $1: 1$ & $89 / 92$ \\
$12^{\mathrm{e}}$ & $\mathrm{CH}_{2} \mathrm{Cl}_{2}$ & $\mathrm{PhCOOH}$ & 95 & $1: 1$ & $90 / 94$ \\
$13^{\mathrm{f}}$ & $\mathrm{CHCl}_{3}$ & PhCOOH & 98 & $1.1: 1$ & $96 / 97$ \\
$144^{\mathrm{g}}$ & $\mathrm{CHCl}_{3}$ & PhCOOH & 51 & $1.1: 1$ & $93 / 94$
\end{tabular}

${ }^{\mathrm{a}}$ Conversion was determined by $1 \mathrm{H}$ NMR spectroscopy. ${ }^{\mathrm{b}}$ Enantiomeric excess was determined by chiral HPLC analysis. ${ }^{c}$ Reaction set up in the glovebox. ${ }^{\mathrm{d}}$ Reaction concentration was $0.5 \mathrm{M}$. ${ }^{\mathrm{e}}$ Reaction concentration was 2 M. ${ }^{\mathrm{f}}$ Reaction heated to $30{ }^{\circ} \mathrm{C}$ in a sand bath for $4 \mathrm{~h} .{ }^{\mathrm{g}}$ Reaction under microwave irradiation $(2 \mathrm{~W})$.

In spite of the excellent levels of enantioselectivity achieved in the reaction, no appreciable levels of diastereoselectivity were recorded in agreement with previous studies. ${ }^{14}$ It has to be noted, however, that the $\gamma$-stereocenter is usually destroyed in subsequent denitration or decarboxlation steps, the stereochemistry at this point being thus irrelevant. ${ }^{14 \mathrm{~b}, \mathrm{c}, 15}$ 


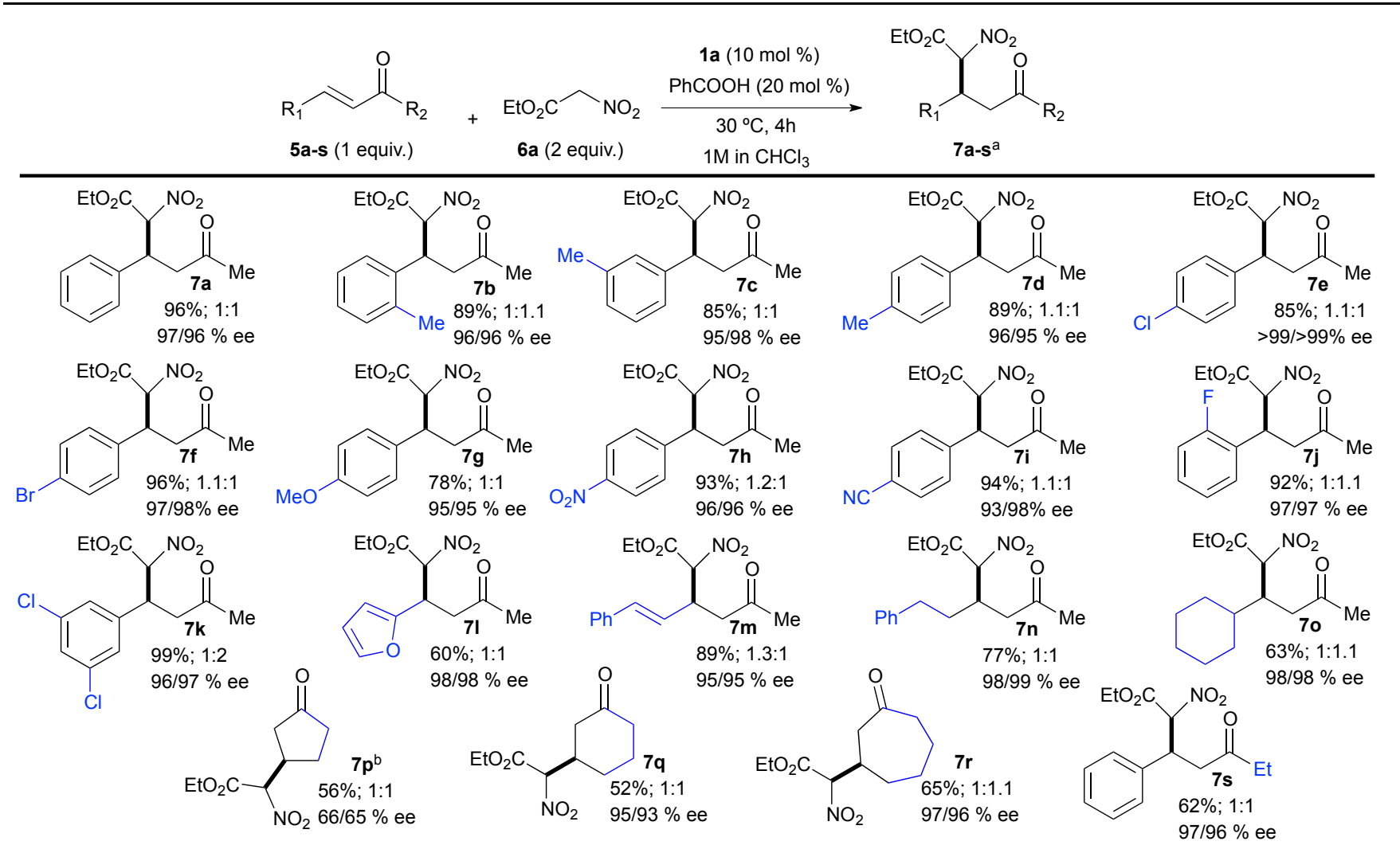

${ }^{\mathrm{a}}$ Isolated yield; diastereomeric ratio and enantioselectivities of each diastereoisomer. ${ }^{\mathrm{b}}$ Cyclopentenone (2 equiv.) and ethyl nitroacetate (1 equiv.) were used in this experiment.

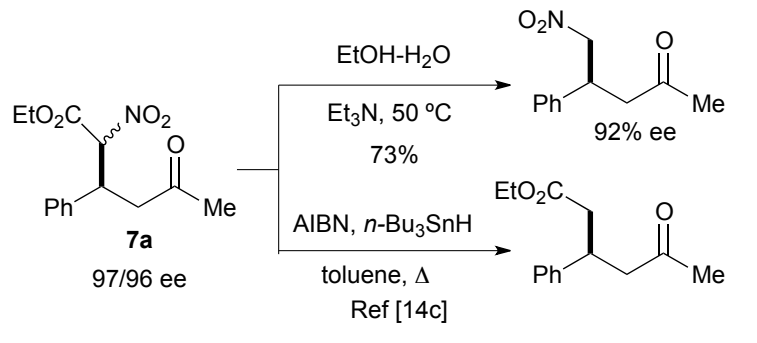

Scheme 2: Decarboxylation and denitration of 7a leading to suppression of the $\gamma$-stereocenter

Once the reaction conditions were optimized, the next step was to explore the scope of the Michael addition of $\mathbf{6 a}$ to a representative family of enones catalized by resin 1a (Table 2). 4-Aryl-substituted 3-buten-2-ones are optimal substrates, irrespectively of the substitution pattern in the aryl group and the electronic characteristics of its substituent (7a-7k). Heteroaryl substituents, as furyl (7l), are well tolerated as well as substrates with extended conjugation that experience regioselective addition to the $\beta$ position (7m). 4-Alkyl substituents (7n and $\mathbf{7 0})$ are also suitable for this transformation, the corresponding additions taking place with excellent enantioselectivity. In the same manner, 1-aryl-1penten-3-one substrates (7s) undergo highly enantioselective addition, but at decreased rate. To our delight, more conformationally constrained systems like cyclic enones are also optimal substrates (7q-r) under catalysis by 1a. Only 2cyclopentenone (7p) shows some limitations, the corresponding addition taking place at slower rate and with lower enantioselectivity.

Next, the applicability of $\mathbf{1 a}$ with different pronucleophiles in the same reaction was tested (Table 3). Gratifyingly, a variety of $\mathrm{C}-\mathrm{H}$ acids underwent Michael addition to a broad range of enones under slightly re-optimized reaction conditions. Bromonitromethane afforded the target Michael adducts with high yield and excellent enantioselectivity by simply increasing catalyst amount to $20 \mathrm{~mol} \%$ and reaction time (8a-e). Malonitrile (9a-c), phenylsulfonylnitromethane (11) and Meldrum's acid (12a-b), which had not been previously used in Michael additions mediated by cinchone-derived amines, were also found to be suitable substrates when treated with an excess of the enone. In a similar manner, $\beta$-keto acid $\mathbf{6 d}$ can be a valuable donor in additions mediated by $\mathbf{1 a}$. The reaction takes place with concomitant decarboxylation, leading to the 1,5dicarbonyl compounds (10a-c). As it can be observed in Table 3 , most of the structural types of enones explored in Table 2 are also suitable for the addition of this broad family of $\mathrm{C}-\mathrm{H}$ acids. 
Table 3. Michael Addition of Different Nucleophiles to Enones.

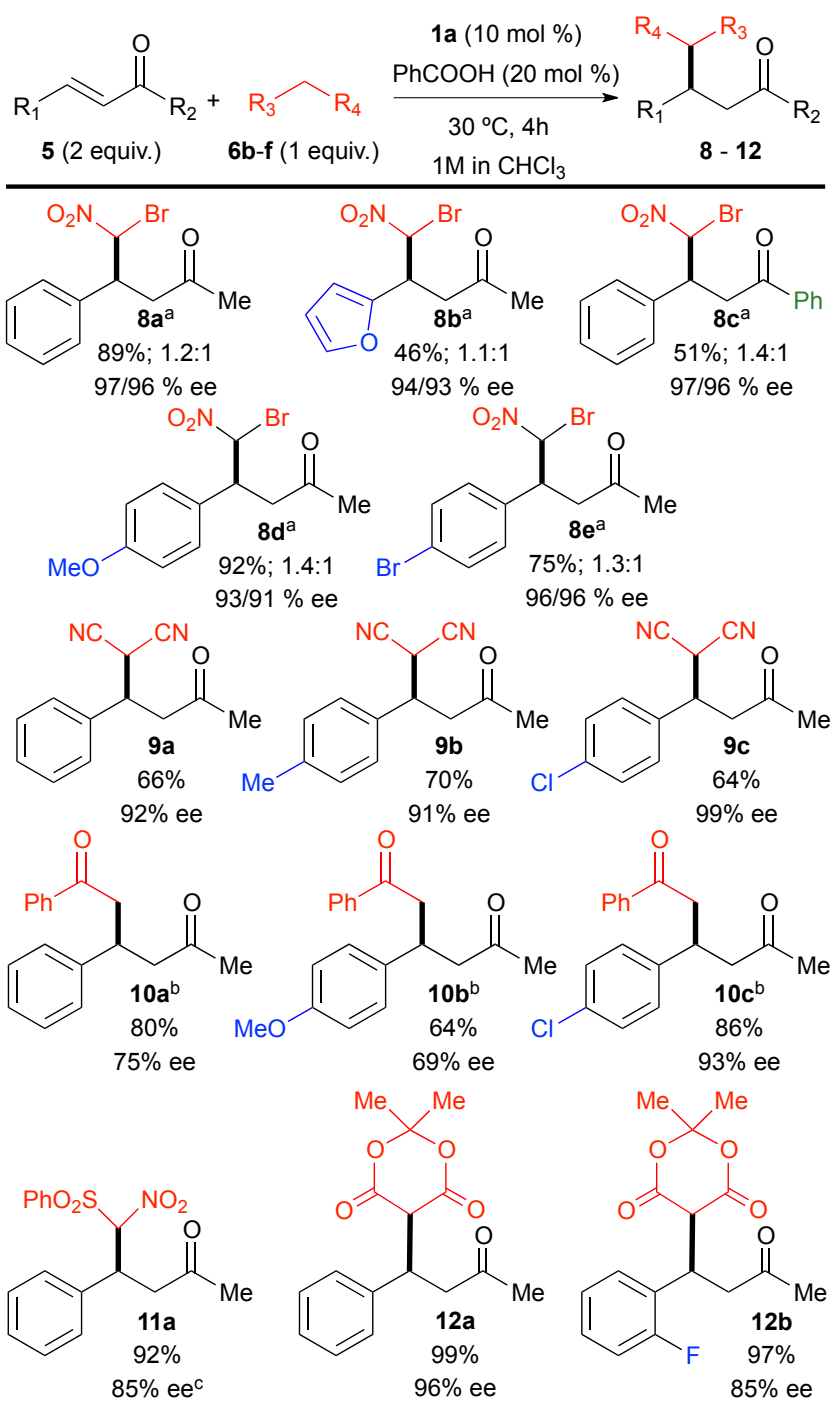

${ }^{a}$ Conditions: 1 (20 mol\%), $\mathrm{PhCOOH}(40 \mathrm{~mol} \%)$, 5a (1 equiv.), bromonitromethane ( 2 equiv.). ${ }^{b} 3$ equiv. of $\beta$-ketoacid and 1 equiv. of enone were used. ${ }^{c}$ Enantiomeric excess was determined on the 1,4-dicarbonyl compound derivative resulting from hydrolysis of the nitrosulfonyl moiety (See ESI $\dagger$ ).

One of the most important advantages offered by immobilized catalysts is the possibility to recover them by simple filtration and reuse in a new catalytic cycle. To assess the robustness of $\mathbf{1 a}$, its recycling in the model Michael addition of ethyl nitroacetate (6a) to the enone (5a) was performed. In six consecutive cycles performed at constant reaction time, no decrease in ee was observed. On the other hand, a slight decrease in conversion between cycles was recorded (see ESI $\dagger$ ).

The high catalytic activity exhibited by $\mathbf{1 a}$ and its robustness convert this catalytic resin into a suitable candidate for a continuous flow processing. The very simple flow reactor used to test this hypothesis involved $450 \mathrm{mg}$ of resin $\mathbf{1}(f=0.87$ mmol. $\left.\mathrm{g}^{-1}\right)$ packed into a Teflon ${ }^{\circledR}$ tube $(1 / 4$ inch i.d.) between two plugs of glass wool. The catalytic reactor was heated to
$30{ }^{\circ} \mathrm{C}$ while a solution of the two reactants (5a and 6a) and benzoic acid in $\mathrm{CHCl}_{3}$ (no reaction occurs in the absence of catalyst) was pumped into the system at $50 \mu \mathrm{l} / \mathrm{min}$ (equivalent to $\approx 40 \mathrm{~min}$ residence time) (see ESI $\dagger$ ). Notably, $3.6 \mathrm{~g}$ (12.9 $\mathrm{mmol}$ ) of $7 \mathbf{a}$ were collected in 21 hours of operation, corresponding to a TON of 32 (Figure 1).

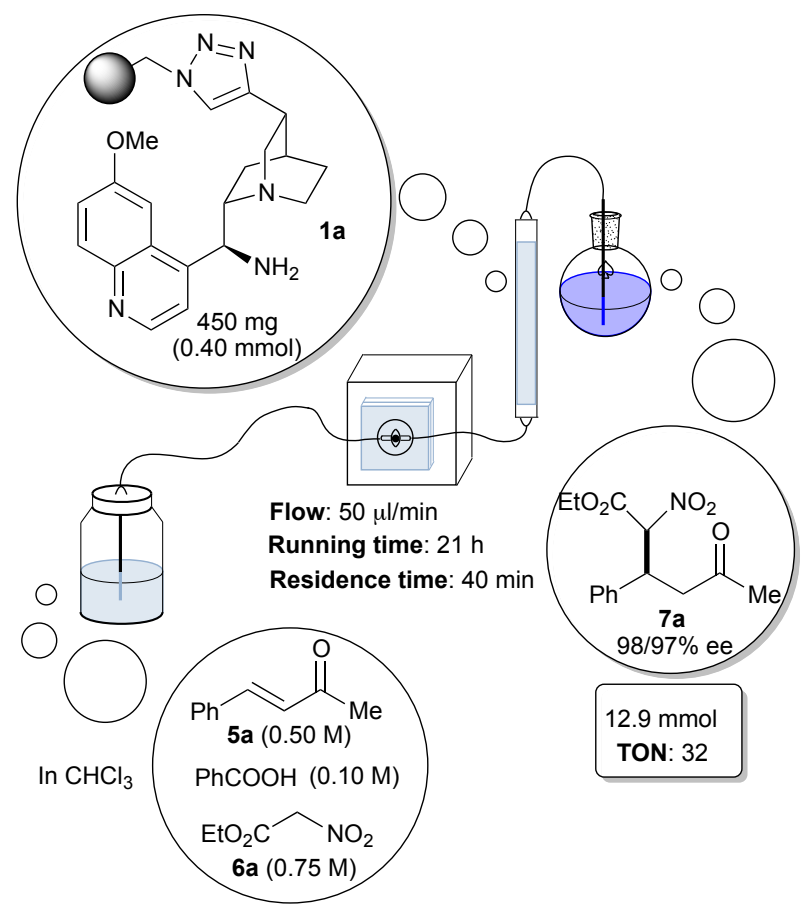

Fig. 1. Continuous Flow Process.

Catalytic asymmetric flow processes based on immobilized catalytic species are of particular interest since focused libraries aimed at lead discovery and optimization can be very easily prepared in this way using a single set-up operated in a sequential manner. ${ }^{11 a, d, 16}$ Taking advantage of the versatility of the PS-supported cinchona aminocatalyst 1a, a diverse library of enantioenriched Michael adducts could be sequentially prepared (Figure 2). Thus, using optimized flow conditions, five Michael adducts $(\mathbf{7 c}, 7 \mathbf{k}, \mathbf{1 1 a}, \mathbf{1 2 a}$, and 12b) were prepared by combination of four different enones with three nucleophiles. Each solution containing the enone, nucleophile and the benzoic acid, was circulated through the system at $50 \mu \mathrm{l} / \mathrm{min}$ for $2 \mathrm{~h}$ (first hour was utilized for stabilization of the flow system whilst only the effluent corresponding to the second hour was collected). For the avoidance of crosscontamination, the column was washed with $\mathrm{CHCl}_{3}$ for $1 \mathrm{~h}$ between the preparation of two consecutive adducts. The complete process takes place with remarkable productivity (TOF $\left.=1.4-3.2 \mathrm{mmol}_{\text {product }} / \mathrm{mmol}_{\text {resin }} \cdot \mathrm{h}\right)$ and affords a library of Michael adducts with increased enantioselectivity in comparison to the corresponding batch processes (Tables 2 and 3). 


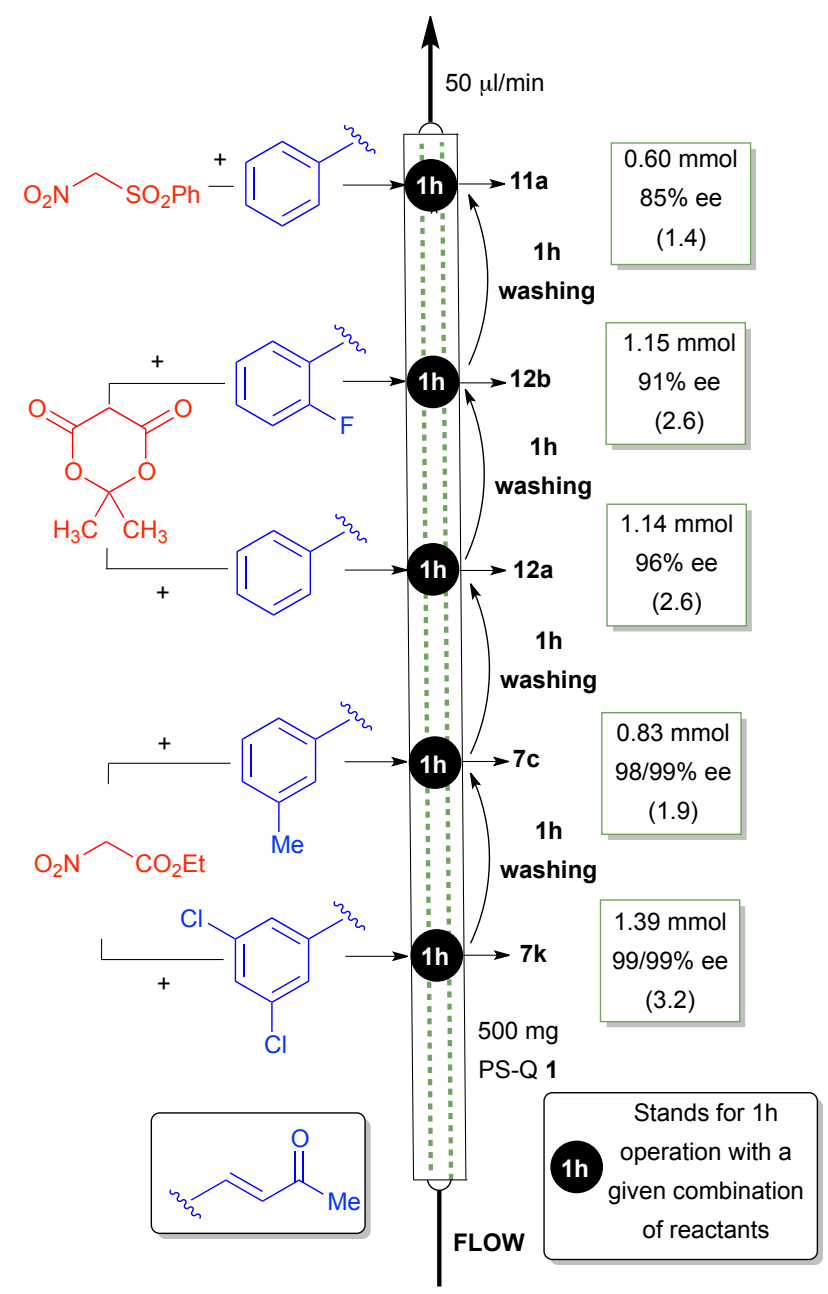

Fig. 2. Continuous Flow Production of a Library of Michael Adducts Catalyzed by Cinchona Alkaloid-Derived 1a. ${ }^{\text {a }}$

${ }^{\mathrm{a}}$ Productivities in $\mathrm{mmol}_{\text {product }} /\left(\mathrm{mmol}_{\text {resin }} \cdot \mathrm{h}\right)$ are shown in parentheses.

\section{Conclusions}

In summary, we have demonstrated that 9-amino(9-deoxy)epi cinchona alkaloids immobilized onto polystyrene resins behave as highly efficient organocatalysts promoting asymmetric Michael additions under batch and flow conditions. Resin 1a has shown high robustness and versatility for the activation of enones towards the addition of C-nucleophiles. Moreover, the suitability of the developed continuous flow process for the sequential preparation of a small library of enantiopure adducts has been demonstrated.

\section{Experimental}

General procedure for the Michael addition of ethyl nitroacetate to enones catalyzed by resin $1 \mathrm{a}$.

Polymer-supported quinine derivative 1a (11.2 $\mathrm{mg}, 0.01 \mathrm{mmol}, 10 \mathrm{~mol} \%)$ was added to a vial with a mixture of $\alpha, \beta$-unsaturated ketone $5(0.1 \mathrm{mmol}$, 1 equiv.), ethyl nitroacetate $\mathbf{6 a}(22 \mu \mathrm{L}, 0.2 \mathrm{mmol}, 2$ equiv.) and benzoic acid (2.4 mg, $0.02 \mathrm{mmol}, 20 \mathrm{~mol} \%)$ in $\mathrm{CHCl}_{3}(0.1 \mathrm{~mL})$ and the mixture was stirred at $30^{\circ} \mathrm{C}$ in a sand bath for $4 \mathrm{~h}$. Then, the resin was filtered off, washed with $\mathrm{CHCl}_{3}(3 \times 1 \mathrm{~mL})$ and dried under vacuum. The combined liquid phases were concentrated under reduced pressure. The reaction crude was directly purified through column chromatography eluting with cyclohexane and (1-20)\% AcOEt.

\section{Acknowledgements}

This work was funded by MINECO (grant CTQ2012-38594C02-01), DEC (grant 2014SGR827), and ICIQ Foundation. We thank MINECO for support through Severo Ochoa Excellence Accreditation 2014-2018 (SEV-2013-0319). J. I. thanks ICIQIPMP-2013 for a fellowship. C. A. thanks MICINN for a Juan de la Cierva fellowship. A. H. H. thanks the MECD for an FPU predoctoral fellowship.

\section{Notes and references}

1. Recent reviews on flow processes: (a) J. Wegner, S. Ceylan, A. Kirschning, Chem. Commun., 2011, 47, 4583; (b) T. Tsubogo, T. Ishiwata, S. Kobayashi, Angew. Chem. Int. Ed., 2013, 52, 6590; (c) A. Puglisi, M. Benaglia, V. Chiroli, Green Chem., 2013, 15, 1790; (d) L. Vaccaro, D. Lanari, A. Marrocchi. G. Strappaveccia, Green Chem., 2014, 16, 3680; (e) J. Hartwig, J. B. Metternich, N. Nikbin, A. Kirschning, S. V. Ley, Org. Biomol. Chem., 2014, 12, 3611.

2. For recent reviews of immobilized catalysts and reagents: (a) J. Lu, P. H. Toy; Chem. Rev., 2009, 109, 815; (b) M. Benaglia. Recoverable and Recyclable Catalysts, Wiley, Chichester, 2009; (c) T. E. Kristensen, T. Hansen, Eur. J. Org. Chem., 2010, 3179.

3. C. E. Song. Cinchona Alkaloids in Synthesis and Catalysis. WileyVCH, Weinheim, 2009.

4. For recent reviews involving 9-amino(9-deoxy)epi cinchone alkaloids: (a) F. Peng, Z. Shao, J. Mol. Catal., 2008, 285, 1; (b) C. Chen, Synlett, 2008. 13, 1919; (c) G. Bartoli, P. Melchiorre, Synlett, 2008, 12, 1759; (d) L. Jiang, Y. C. Chen, Catal. Sci. Technol., 2011, 1, 354; (e) L. W. Xu, J. Luo, Y. Lu, Chem. Commun., 2009, 1807; (f) P. Melchiorre, Angew. Chem. Int. Ed., 2012, 51, 9748.

5. (a) P. Yu, J. He, C. Guo, Chem. Commun., 2008, 2355; (b) P. GarcíaGarcía, A. Zagdoun, C. Copéret, A. Lesage, U. Díaz, A. Corma, Chem. Sci., 2013, 4, 2006.

6. J. W. Lee, T. Mayer-Gall, K. Opwis, C. E. Song, J. S. Gutmann, B. List, Science, 2013, 341, 1225.

7. O. Gleeson, G. L. Davies, A. Peschiulli, R. Tekoriute, Y. K. Gun'ko, S. J. Connon, Org. Biomol. Chem., 2011, 9, 7929.

8. Some examples of cinchona alkaloids and derivatives polymerized: (a) M. Inagaki, J. Hiratake, Y. Yamamoto, J. Oda, Bull. Chem. Soc. Jpn., 1987, 60, 4121; (b) S. H. Youk, S. H. Oh, O. S. Rho, J. E. Lee, J. W. Lee, C. E. Song, Chem. Commun., 2009, 2220; (c) G. M. Miyake, H. Ida, H. Y. Hu, Z. Tang, E. Y. X. Chen, E. Yashima, J Pol. Sci. Part A., 2011, 49, 5192; PEG-supported (d) B. Thierry, J. C. Plaquevent, D. Cahard, Tetrahedron: Asymmetry, 2003, 14, 1671; (e) X. Wang, L. Yin, T. Yan, Y. Wang; Tetrahedron: Asymmetry 2007, 18, 108; PS-supported: (f) B. Thierry, J. C. Plaquevent, D. Cahard, Tetrahedron: Asymmetry, 2001, 12, 983; (g) R. Chinchilla, P. Mazón, C. Nájera; Adv. Synth. Catal., 2004, 346, 1186. 
9. K. A. Fredriksen, T. E. Kristensen, T. Hansen, Belstein, J. Org. Chem., 2012, 8, 1126.

10. (a) A. M. Hafez, A. E. Taggi, T. Dudding, T. Lectka, J. Am. Chem. Soc., 2001, 123, 10853; (d) F. Bonfils, I. Cazaux, P. Hodge, C. Caze, Org. Biomol. Chem., 2006, 4, 493.

11. For recent examples: (a) P. Kasaplar, C. Rodríguez-Escrich, M. A. Pericàs, Org. lett., 2013, 15, 3498; (b) X. Fan, C. Rodríguez-Escrich, S. Sayalero, M. A. Pericàs, Chem. Eur. J., 2013, 19, 10814; (c) A. H. Henseler, C. Ayats, M. A. Pericàs, Adv. Synth. Catal., 2014, 356, 1795; (d) C. Ayats, A. H. Henseler, E. Dibello, M. A. Pericàs, $A C S$ Catal., 2014, 4, 3027. For examples involving cinchone alkaloids immobilization: (e) K. M. Kacprak, N. M. Maier, W. Lindner. Tetrahedron lett., 2006, 47, 8721; (f) R. P. Jumde, A. Di Pietro, A. Manariti, A. Mandoli. Chem. Asian, J., 2015, 10, 397.

12. (a) J. Frackenpohl, W. M. Braje, H. M. R. Hoffmann, J. Chem. Soc., Perkin Trans. 1, 2001, 47; (b) S. Röper, M. H. Franz, R. Wartchow, H. M. R. Hoffmann, Org. Lett., 2003, 5, 2773.

13. (a) J. Comelles, M. Moreno-Mañas, A. Vallribera, Arkivoc, 2005, 9, 207; (b) J. Christoffers, G. Koripelly, A. Rosiak, M. Rössle, Synthesis, 2007, 9, 1279; (c) J. L. Vicario, D. Badía, L. Carrillo, Synthesis, 2007, 14, 2065; (d) S. Sulzer-Mossé, A. Alexakis, Chem. Commun. 2007, 3123; (e) S. B. Tsogoeva, Eur. J. Org. Chem., 2007, 1701; (f) D. Almasi, D. A. Alonso, C. Nájera, Tetrahedron: Asymmetry, 2007, 18, 299; (g) S. Mukherjee, J. W. Yang, S. Hoffmann, B. List, Chem. Rev., 2007, 107, 5471; (h) C. Bhanja, S. Jena, S. Nayak, S. Mohopatra, Belstein, J. Org. Chem., 2012, 8, 1668.

14. (a) C. Liu, Y. Lu, Org. Lett., 2010, 12, 2278; (b) H. W. Moon, D. Y. Kim, Tetrahedron lett., 2010, 51, 2906; (c) H. W. Moon, D. Y. Kim, Bull. Korean. Chem. Soc., 2011, 32, 291.

15. In our hands, the decarboxylation of compound $7 \mathbf{a}$ afforded the corresponding $\gamma$-nitroketone in $73 \%$ isolated yield and $92 \%$ ee. (See ESI†).

16. (a) M. A. Pericàs, C. I. Herrerías, L. Solà, Adv. Synth. Catal., 2008, 350, 927; (b) C. Ayats, A. H. Henseler, M. A. Pericàs, ChemSusChem 2012, 5, 320; (c) L. Osorio-Planes, C. Rodríguez-Escrich, M. A. Pericàs, Chem. Eur. J., 2014, 20, 2367. 